\title{
Communication in Discrete Action-Response Games
}

\author{
Peter L. Hurd \\ Division of Ethology, Department of Zoology, University of Stockholm, S-106 91 Stockholm, \\ Sweden
}

(Received on 8 July 1994, Accepted in revised form 21 November 1994)

\begin{abstract}
I present a simple game, the Basic Action-Response game, which allows investigation of the claim that signals must be costly to be reliable. The Basic Action-Response game is the simplest communication game possible, by investigating its parameters we are able to define clearly "conflict", "handicap", "communication" and other relevant concepts. I explore the conditions on the magnitude of the stabilizing cost and handicap that must hold in order to maintain the evolutionary stability of signalling. It will be demonstrated that stable communication need not make use of costly signals at ESS, not even "on average", and that "negative handicaps" can be stable as long as the stabilizing cost is large enough.
\end{abstract}

\section{Introduction}

It is widely considered a truism in biology today that signals must be costly to be reliable. This view stems from the so-called handicap principle first proposed by Amotz Zahavi nearly 20 years ago (Zahavi, 1975, 1977). The most common interpretation of Zahavi's handicap notion is that the form of a signal must be intrinsically costly for communication to exist. The handicap hypothesis was long looked upon with great suspicion until given concrete interpretation by Grafen (1990).

Current attempts to understand the evolution of communication began with the revitalizing of evolutionary thinking of the late sixties and seventies. With the introduction of evolutionary game theory it became clear that communication was not a simple issue. That signalling can be evolutionarily stable has been demonstrated by such theoretical work as Enquist (1985), Pomiankowski (1988) and Grafen (1990) and classical game theory (often ignored by biologists) has reached similar conclusions (Spence, 1973; Crawford \& Sobel, 1982; Kreps \& Wilson, 1982; Milgrom \& Roberts, 1982).

Grafen (1990: 521) derived three separate "main handicap results" about evolutionarily stable signalling, they are: (a) that the signaller uses different signals in different states (i.e. that information is made available to the receiver, or that something is "honestly" signalled);

(b) that signals are costly;

(c) that they costlier for worse signallers.

The first condition, combined with a receiver that responds to this variable signal, defines communication. The third condition deals with "preventing cheating". It tells us that if communication is an ESS some individuals must prefer to use a signal which elicits a less preferred response from the receiver. The third condition is the key to understanding signalling in situations without complete common interest between the players (Enquist, 1985).

It is the second condition that has spawned much recent debate (Maynard Smith, 1991; Grafen \& Johnstone, 1991).

I investigate the handicap hypothesis within the minimum game capable of describing signalling, the Basic Action-Response Game. I shall explore the conditions that must hold in order to maintain the evolutionary stability of signalling, making particular reference to Grafen's three conditions.

(C) 1995 Academic Press Limited 


\section{The Basic Action-Response Game}

The Action-Response Game is very simple. One individual, the Signaller, S, observes some variable state and then acts, a second individual, the Receiver, $\mathrm{R}$, observes this act and then responds. The game is then over. In the most simple version of this game, referred to here as the Basic Action-Response game, there are only states, two possible actions, and two possible responses. In classical game theory this game is sometimes known as the basic communication game (e.g. Fudenberg \& Tirole, 1991).

The game is described in Fig. 1 using the extensive form. While evolutionary biologists are most familiar with games in matrix form, matrices are not always the best way of describing games and several alternatives

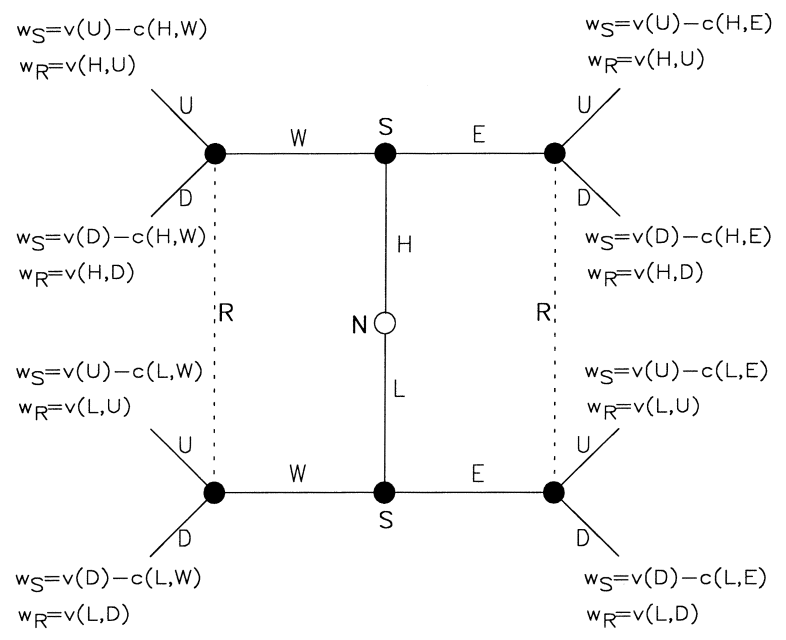

FIG. 1. The basic Signal-Response game is described using the extensive form. The root of the tree, indicated by an open circle, is the start of the game, in this case the root is in the middle of the tree. Other nodes, the filled dots, describe different states of the game as play progresses. Branches in the tree describe alternative moves by the players and by nature (a bold letter next to a node indicates which player moves at that node). The first move is a move-by-nature, $\mathrm{N}$, and is either to the High or Low branch. This is the state which is observed by the Signaller, S. Moves by nature occur according to some probability distribution and are not the product of any strategy or player control (it can be assumed for pedagogical reasons that High and Low have equal probability, but the actual value doesn't influence the nature of the solutions at all). Branches further out in the tree represent subsequent possible moves by players. After observing the result of the move-by-nature, $\mathrm{S}$ chooses to do either the East or West behaviour. In the last stage of the game, player R replies by choosing between the Up and Down responses.

Also indicated in the tree are information sets and payoffs. An information set is a collection of nodes between which a player is not capable of discriminating, these sets are indicated by joining member nodes with a dotted line. Note that in this figure player R's information sets separate states in which player $\mathrm{S}$ has chosen to play East and West, but not the move-by-nature separating High from Low. This means that while player S knows whether the game is in the High or Low state, player R does not, but player R knows what move player $\mathrm{S}$ has made before having to choose his (or in our favourite interpretation, her) reply. Payoffs for any given route through the tree are noted at the end of the last branch. do exist. There are several reasons why game theorists often prefer the extensive form. Most importantly is that it allows a sequence of actions or decisions through time to be analyzed in a clear and well-defined way. If the game is not too complicated it can be drawn, as shown in Fig. 1.

To make the game relevant in terms of communication, we must assume that the initial "move-bynature" is hidden from the receiver. The receiver does not know the state of the game but does know which behaviour the signaller has used before having to choose his (or in our favourite interpretation, her) reply. In the extensive form this means that the state of the receiver cannot be specified with single node. A collection of nodes between which a player is not capable of discriminating is called an information set, these sets are indicated by joining member nodes with a dotted line. Note that in Fig. 1, the receiver's information sets distinguish between states in which player the signaller has chosen to play West and East, but not the move-by-nature separating High from Low. The move-by-nature makes $\mathrm{S}$ either a High state or a Low state signaller. Also indicated in Fig. 1 is the payoff received by the players for any given route through the tree.

We will not solve the general case here, but will instead simplify the problem with the following assumptions. Receiver fitness is a function of the state, $z$, and the receiver response, $r$,

$$
w_{R}=v(z, r) .
$$

The signaller fitness is a sum of the value of the receiver response, $r$, and the intrinsic cost of the signal, $c$, which depends on state $z$ and the signaller behaviour, $s$,

$$
w_{S}=v(r)-c(z, s) \text {. }
$$

We will consider pure strategies contingent upon all information available to the player. Strategies for the actor prescribe an action as a function of the initial state of the game, High or Low. Similarly strategies for the receiver prescribe a response to make as a function of player S's action. So the signaller's strategies are of the form $\mathrm{S}$ (what to do if state is High, what to do if state is Low), and the receiver's are R (what to do if S plays West, what to do if S plays East). A pure strategy for the signaller must be one of the following $(E, E)$, $(E, W),(W, E)$ and $(W, W)$. Likewise the receiver's strategy must be one of $(U, U),(U, D),(D, U)$ and $(D, D)$.

In the Basic Action-Response game each player has four possible pure strategies for each player, giving rise to 16 possible pairings. Communication (sensu Enquist et al., 1985) occurs in only four of these strategy pairings, when player $S$ provides a variable signal, and 
the receiver's response is contingent upon that signal. However these four cases are formally identical and we need only consider one of them, $(W, E)$ and $(U, D)$. All the ESS solutions considered will use this pair of strategies.

\section{The Signalling Solutions}

Although superficially simple, this game provides a number of different communication solutions resulting from different payoff patterns to the signaller and receiver. We will examine the properties of different communication ESSs resulting from different payoff patterns, starting with the payoffs to the receiver.

\subsection{RECEIVED PAYOFFS}

For communication to occur it must pay the receiver to respond differently to High and Low state signallers, accepting the former with an Up reply, and rejecting the latter with a Down. This is the only assumption we will make about the reactor's payoffs, and it means that the following must be true,

$$
\begin{gathered}
w_{R}(H, U)>w_{R}(H, D), \\
w_{R}(L, D)>w_{R}(L, U) .
\end{gathered}
$$

\subsection{SIGNALLER BENEFITS}

We will assume that the signaller would always prefer that the receiver use the Up response. This puts the signaller and receiver in conflict, since the receiver prefers to turn Down Low state signallers:

$$
v(U)>v(D) .
$$

We define the relative value of an Up response to the signaller as

$$
V=v(U)-v(D)>0 .
$$

$V$ is equal for all signallers, combined with (1) this means that signallers are indicating their quality rather than their need.

\subsection{SIGNALLER COSTS}

In order for communication to exist there must be a variable signal. The Basic Action-Response Game strategies which lead to a variable signal are $(W, E)$ and $(E, W)$. We have assumed $(W, E)$. This means $W$ is the behaviour which elicits the preferred response from the receiver. This variable signal from $\mathrm{S}$ matched with the variable reply from $\mathrm{R}$ fulfils Grafen's result (a). In the context of this game Grafen's result (a) and communication are synonymous.
In order for $(W, E)$ to be the unique, best reply to $(U, D)$, it must be the case that,

$$
\begin{gathered}
v(U)-c(H, W)>v(D)-c(H, E) \\
v(D)-c(L, E)>v(U)-c(L, W)
\end{gathered}
$$

Since $W$ is the behaviour eliciting the preferred reply, we will define the relative cost of behaviours in terms of the cost of this one. $C_{H}$ is the cost of behaviour $W$ to a High state signaller and $C_{L}$ is the cost to a low state signaller,

$$
\begin{aligned}
C_{H} & =c(H, W)-c(H, E), \\
C_{L} & =c(L, W)-c(L, E) .
\end{aligned}
$$

It follows from inequality (4) that,

$$
C_{L}>V>C_{H} .
$$

\subsection{GRAFEN'S RESULTS (a) \& (c)}

Grafen's result (c) requires that the behaviour which would elicit the preferred response from the signaller is "costlier for worse males", Grafen (1990). From inequality (7) we see that $C_{L}$ is greater than $C_{H}$. In other words, Grafen's (c) also follows directly from the definition of communication.

All versions of this game can be plotted as a function of the parameters $C_{H}$ and $C_{L}$ for any given $V$. Figure 2 shows this solution space as defined by inequality (7).

We have demonstrated that Grafen's (1990) (a) and (c) results are required for stable communication under conditions of conflict. We next consider whether result (b) must be true.

\subsection{GRAFEN'S RESULT (b)}

Grafen's result (b) states that the signal is costly. For our purposes, Grafen's result (b) means that $C_{H}$ is positive. Note that when an ESS strategy pair is in use, only the High-West-Up and Low-East-Down outcomes will ever be seen. The only $W$ behaviours used are those of the High state signallers, the cost to them of the behaviour is $C_{H}$, this is traditionally referred to as the handicap cost. However, so far, nothing can be said about the sign of $C_{H}$. Two cases can exist in which Grafen's (b) is either true or not true. If the stabilizing, $C_{L}$, cost is large enough then the handicap can be negative - an "anti-handicap" - and remain a communication ESS just as valid as that of the standard handicap model (Fig. 2). Whether the "anti-handicap", (b)-violating cases can really be called signalling is debatable, since the receivers behaviour would not change if the information constraint were removed from the game. This point will be addressed further in context of the definition of signal in the conclusion. Regardless, the conflict between signaller and receiver 


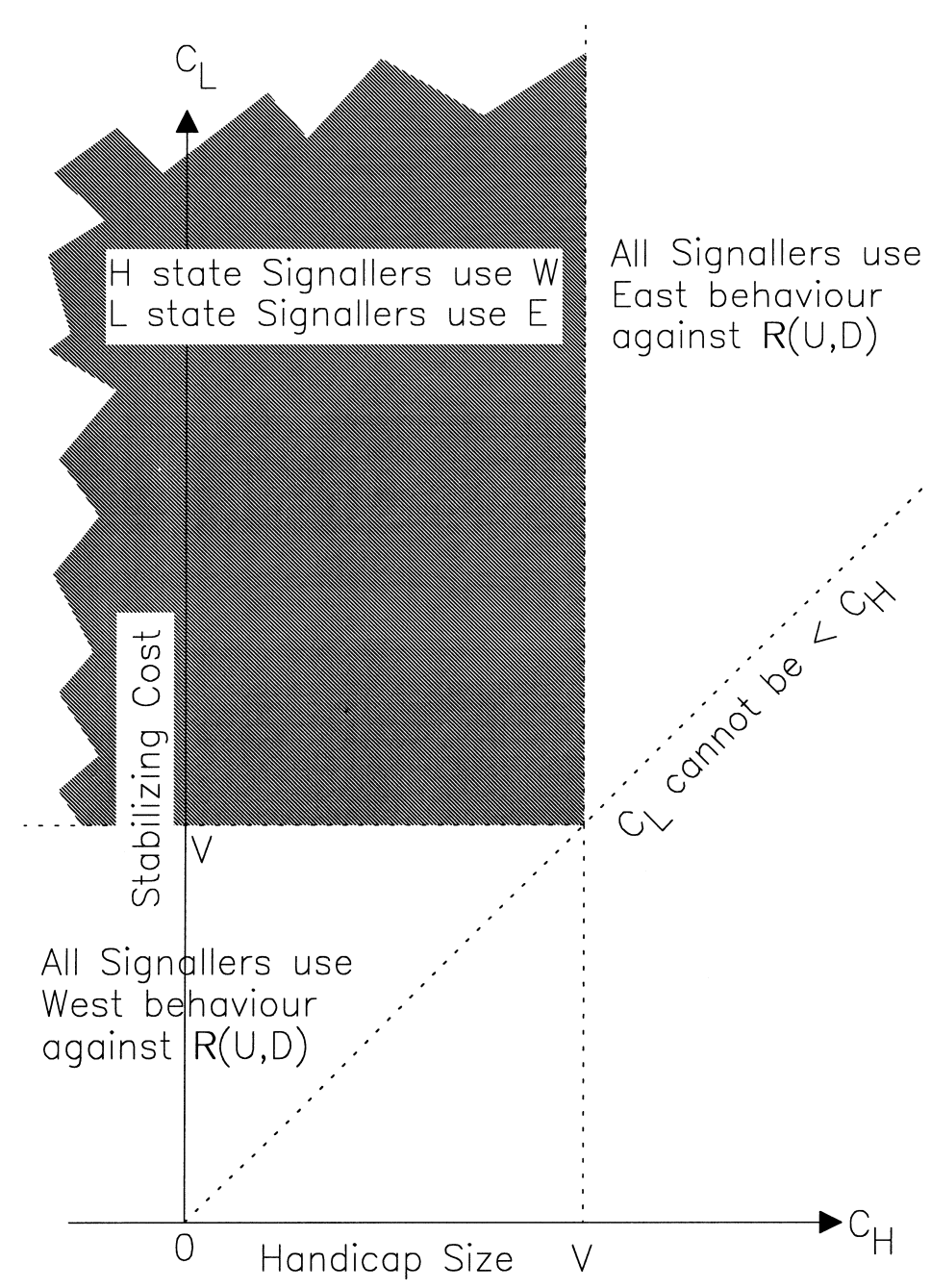

FIG. 2. A pair of cost parameters, $\left(C_{H}, C_{L}\right)$ specifies point in the plane of all possible versions of this game for any particular $V$. The lines $C_{H}=V$ and $C_{L}=V$ divide the plane into four different areas around the point $(V, V)$. In the shaded area signalling is an $\mathrm{ESS}$, there $\mathrm{S}(W, E)$ is the best reply to $\mathrm{R}(U, S)$.

still exists, and communication still occurs, furthermore no specific signal need evolve to indicate signaller state.

In addition to negative handicapped signalling, Fig. 2 demonstrates a space below the $C_{L}=V$ line where the handicap is still positive, but the cost to Low signallers is not large enough to preserve communication. The handicap alone will not ensure communication will be stable.

\section{Biological Examples}

To make our results easier to follow, consider the following biological examples. The most familiar in terms of the handicap debate is the mate choice game, where the High move-by-nature means that the prospective suitor $\mathrm{S}$, is a high quality mate, and the
Low move-by-nature means that the suitor is of low quality, a sub-adult male perhaps. The signaller's West behaviour means making some display of male quality, for example growing a green plumage. The receiver, a courted female, accepts the suitor with an Up behaviour (picks him up, so to speak) and refuses with a Down behaviour (i.e. turns him down).

Another interpretation is predator signalling. The signaller has either detected or not detected a predator, the move by nature. The act is then either to make some signal of having detected the predator (or the non-predator if none is detected), or to continue without signalling. The receiver, a predator, then either continues to stalk the prey, or abandons the hunt.

A simplistic biological example of the "antihandicap" ESS case would be a male insectivorous bird, which catches prey on the wing. If high-quality 
males can grow long tail feathers at essentially no cost, and low quality males cannot, then females will prefer long tailed mates. If one assumes that long tails are aerodynamically advantageous (Jennions, 1993), then a stable signalling system can still exist. This requires that low-quality males benefit by doing something other than growing long tail feathers, such as allocating more resources to the immune system (Wedekind \& Folstad, 1994).

\section{Conclusion}

We have considered the simplest communication game possible - the Basic Discrete Signal-Response game. Even in the most conservative situation, the mechanisms producing stable signalling do not universally produce costly signals. The handicap costs can be reduced to zero and beyond, while communication continues to be stabilized by costs that would be paid by invading exploitative strategies though the stabilizing cost. It is the discrete nature of this game that allows us to separate the handicap from the stabilizing cost. The cost-free signalling described here is unlikely to be found in situations where states and signals are continuous.

Throughout this paper I have used the term "signal" to refer to any stimulus which transmits information. In biology, this word is traditionally defined as a trait which has specifically evolved for the purposes of communication. In contrast, a "cue" is a trait, perhaps costly, not specifically evolved for the purposes of communication, but which is used by receivers as a source of information (Markl, 1985). Given this definition, communicating stimuli with negative handicaps ought perhaps to be called cues, while those with zero and positive handicaps may be either cues or signals. It is possible that a communicating stimulus has evolved for the purpose of communication yet has a handicap cost of zero (e.g. aposematic colouration), given that one hasn't defined "communication" as the use of inherently costly signals (e.g. Hasson, 1994). Using the word signal to mean "stimulus providing information" (in contrast to the traditional definition of "noise") prevents "costly signalling" from being a tautology, as seems to be the case with the current terminology.

No signalling costs at all need be paid by any individuals when the game is at a signalling ESS. In more biological terms, mutants who do not play the ESS strategy are the only ones which must pay "the cost of honesty". Hardly a surprising conclusion, given the general idea of an ESS as a strategy which it does not pay to deviate from. This is contrary to the conclusions of Grafen (1990, Johnstone \& Grafen,
1993) and Zahavi (1993). "If we see a character which does signal quality, then it must be a handicap" (Grafen, 1990). Our "anti-handicap" finding does not support this statement. If the stabilizing cost is large enough, then a negative handicap can be used to signal quality.

However, this does not mean that all signals will not be costly. In fact, it seems likely that the majority of games with handicapped signalling will yield predictions about costly signals, for example as in Grafen's model. Zahavi's idea can be transformed from a principle to a specific mechanism whereby stable communication can be maintained. This has the advantage of creating some understanding about the evolutionary mechanisms underlying signalling. If we consider communication without limiting ourselves to classically handicapped cases, then we are likely to find that cost-free conventional signals, and negative cost "anti-handicap" communication often exist. It seems very clear that some signals which even occur in conflict situations (e.g. facial expressions in primates and carnivores) are not costly. Although cost is an integral part of most games, this does not mean that they are all necessarily handicapped games. All games are at base cost/benefit optimizations - to call all costs "handicaps" merely because they relate to a communication game is a bit pointless.

I wish to thank an anonymous reviewer and John Maynard Smith for helpful comments on the manuscript. Magnus Enquist provided guidance throughout the development of the game and manuscript. Olof Leimar seeded the ideas and gave valuable input and discussion. This work was supported by an NSERC Canada PGS, and by a Swedish Natural Science Research Council grant to Magnus Enquist.

\section{REFERENCES}

Crawford, V. \& Sobel, J. (1982) Strategic information transmission. Econometrica. 50, 1431-1452.

ENQUIST, M. (1985) Communication during aggressive interactions with particular reference to variation in choice of behaviour. Anim. Behav. 33, 1152-1161.

Enquist, M., Plane, E. \& Roed, J. (1985) Aggressive communication in fulmars (Fulmarus glacialis) competing for food. Anim. Behav. 33, 1007-1020.

FudenberG, D. \& Tirole, J. (1991) Game Theory. Cambridge, MA: MIT Press.

Grafen, A. (1990) Biological signals as handicaps. J. theor. Biol. 144, 517-546.

Hasson, O. (1994) Cheating signals. J. theor. Biol. 167, 223-238.

JENnions, M. D. (1993) Female choice and the cost of long tails. Trends Ecol. Evol. 8, 230-232.

Johnstone, R. A. \& GrafEn, A. (1991) The continuous Sir Philip Sidney game: a simple model of animal signalling. J. theor. Biol. 156, 215-234.

Johnstone, R. A. \& Grafen, A. (1993) Dishonesty and the handicap principle. Anim. Behav. 346, 759-764.

KREPS, D. \& Wilson, R. (1982) Reputation and imperfect information. J. Econ. Theor. 27, 253-279. 
MARKL, H. (1985) Manipulation, modulation, information, cognition: some of the riddles of communication. In: Experimental Behavioural Ecology and Sociobiology (Holldobler, B. \& Lindauer M., eds) pp. 163-194. Sunderland, MA: Sinauer.

Maynard Smith, J. (1991) Honest signalling: the Phillip Sidney game. Anim. Behav. 42, 1034-1035.

Milgrom, P. \& Roberts, J. (1982) Predation, reputation, and entry deterrence. J. Econ. Theor. 27, 280-312.

Pomiankowski, A. N. (1988) The evolution of female preferences for male genetic quality. Oxford Surveys Evol. Biol. 5, 136-184.
Spence, A. M. (1973) Job market signalling. Q. Jl Econ . 90, 225-243 WedeKind, C. \& Folstad, I. (1994) Adaptive or nonadaptive immunosuppression by sex hormones? Am. Nat. 143, 936-938.

ZAHAVI, A. (1975) Mate selection - a selection for a handicap. J. theor. Biol. 53, 205-214.

ZAHAVI, A. (1977) Reliability in communication systems and the evolution of altruism. In: Evolutionary Ecology (Stonehouse, B. \& Perrins, M. C., eds) pp. 253-259. London: Macmillan.

ZaHavi, A. (1993) The fallacy of conventional signalling. Phil. Trans. R. Soc. Lond. B 340, 227-230. 